\title{
Cerebral micro-embolization during pulmonary vein isolation: Relation to post-ablation silent cerebral ischemia
}

\author{
Edina Nagy-Baló ${ }^{1}$, Mihran Martirosyan ${ }^{1}$, Gábor Sándorfi ${ }^{1}$, Orsolya Hajas ${ }^{1}$, Levente Lánczi ${ }^{2}$, \\ Ervin Berényi ${ }^{2}$, Lilla Ladányi ${ }^{2}$, Alexandra Kiss ${ }^{1}$, István Édes ${ }^{1}$, Zoltán Csanádi ${ }^{1}$ \\ ${ }^{1}$ Department of Cardiology, University of Debrecen, Hungary \\ ${ }^{2}$ Department of Radiology, University of Debrecen, Hungary
}

\begin{abstract}
Background: High incidences of silent cerebral ischemia (SCI) have been revealed by diffusion-weighted magnetic resonance imaging (DW MRI) after pulmonary vein isolation (PVI) for atrial fibrillation. A high number of mostly gaseous micro-embolic signals (MESs) was detected by transcranial Doppler (TCD) during PVI. In this investigation the possible relationship between MESs detected intraoperatively by TCD and new SCI on DW MRI post-ablation is reported.

Methods: 27 consecutive atrial fibrillation patients (6 female, age median: 64 interquartile range: 13.23) undergoing PVI with the pulmonary vein ablation catheter, pre- and post-ablation DW MRI and intraoperative MES detection by TCD were included in the study. Procedures were performed on a therapeutic international normalized ratio (2-3) and with a target activated clotting time $\geq 350 \mathrm{~s}$ in all patients. $D W$ MRI scans performed pre-and post-ablation revealed new SCI in 6 out of 27 (22\%) patients.

Results: The median (interquartile range) MES count recorded during the whole procedure was 1642 (912) in patients with and 1019 (529) in those without SCI $(p=0.129)$. The number of MESs recorded during pulmonary vein angiography was significantly higher in patients as compared to those without a new lesion on the post-ablation DW MRI: 257 (249) vs. 110 (71), respectively ( $p=0.0009)$. On multivariate logistic regression, the total MES count was predictive of SCI in patients older than 68 years. Conclusions: Micro-embolus generation detected by TCD during pulmonary vein angiography signifcantly correlates with new SCI on DW MRI post-ablation. (Cardiol J 2017; 24, 3: 234-241)
\end{abstract}

Key words: silent cerebral ischemia, diffusion-weighted magnetic resonance imaging, transcranial Doppler, microembolic signals

\section{Introduction}

Silent cerebral ischemia (SCI) observed on diffusion-weighted magnetic resonance imaging (DW MRI) as a complication of atrial fibrillation (AF) ablation has been reported at varying rates [1-7] and generated significant concern regarding the long-term consequences of these procedures. The diagnosis of SCI is based on the comparison of two cerebral DWI MRI examinations, usually performed 48-72 $\mathrm{h}$ apart with the AF ablation procedure carried out between them. This imaging modality provides no intra-procedural data which would suggest the mechanism of lesion formation. In contrast, bilateral insonation of the middle cerebral artery (MCA) using transcranial Doppler (TCD) offers a real-time assessment of micro-embolization throughout the whole procedure. The use of TCD during AF ablation was first reported by Kilicaslan et al. [8], who found a significant

Address for correspondence: Zoltan Csanádi MD, PhD, DSc, FESC, Department of Cardiology, University of Debrecen, 22 Móricz Zs., Debrecen, Hungary, tel: +36 209277 231, H-4032, e-mail: drcsanadi@hotmail.com

Received: 12.09.2016

Accepted: 23.11.2016 
relationship between the number of micro-embolic signals (MESs) on TCD and clinical stroke events. The present group has demonstrated that the majority of MESs are gaseous and the generation of micro-emboli is concentrated in the phase of energy delivery with some ablation techniques $[9,10]$. Further, the site of ablation, the ongoing rhythm and certain biophysical parameters during phased radiofrequency $(\mathrm{RF})$ ablation have been found to be predictive of the rate of microembolization [11, 12].

This was prospectively investigated and the rate of micro-embolization during pulmonary vein isolation (PVI) and the incidence of new postablation cerebral lesions on DW MRI in patients undergoing PVI with phased RF and the pulmonary vein ablation catheter (PVAC) was reported.

\section{Methods}

\section{Study population}

Consecutive patients undergoing PVI with the PVAC and the GENius TM 14.4 RF generator for symptomatic paroxysmal or persistent, but not long-standing persistent AF not adequately controlled by at least one antiarrhythmic drug were considered for enrollment in the study. All these patients were asked to consent to continuous, bilateral TCD detection during the ablation procedure, and to DW MRI examinations before and after PVI. Standard exclusion criteria including hyper- or hypothyroidism, heart failure, left atrial (LA) diameter exceeding $50 \mathrm{~mm}$ and previous clinical ischemic stroke or transient ischemic attack were applied. The study design conformed with the guiding principles of the Declaration of Helsinki, and was approved by the Institutional Ethics Committee.

\section{Ablation procedure}

Patients were hospitalized 1 or 2 days before the procedure. Transesophageal echocardiography was carried out within $24 \mathrm{~h}$ prior to the procedure in order to rule out the presence of a cardiac thrombus. All procedures were performed on uninterrupted vitamin $\mathrm{K}$ antagonist therapy with a target international normalized ratio of 2.0 to 3.0, which was confirmed on the day of the ablation. The ablation protocol was reported earlier [10-12]. In brief, under conscious sedation, decapolar and quadripolar catheters were advanced from the femoral vein and positioned in the coronary sinus and the right ventricle. Immediately before transseptal puncture, a $150 \mathrm{IU} / \mathrm{kg}$ body weight i.v. heparin bolus was given, followed by a continuous infusion and additional 2000-5000 IV i.v. boluses of heparin were administered if needed to reach a target activated clotting time (ACT) level of $350 \mathrm{~s}$. The ACT was always checked prior to the first ablation and every 20 min thereafter. RF delivery was not started until the ACT reached at least $300 \mathrm{~s}$. A single trans-septal puncture was performed under fluoroscopic and intra-cardiac echocardiographic guidance, using a standard technique and an $8.5 \mathrm{~F}$ SL0 Swartz sheath (St Jude Medical, Minnetonka, MN, USA). This sheath was then exchanged for a deflectable $65-\mathrm{cm}$-long sheath with a $12-\mathrm{Fr}$ inner lumen (FlexCath, Medtronic CryoCath LP, Kirkland, Quebec, Canada) to be used as a guide for the PVAC (Ablation Frontiers, Medtronic, Carlsbad, CA, USA). The distal circular segment of the PVAC was captured under saline before introduction into the sheath in order to eliminate any microbubbles possibly entrapped between the electrode arrays.

The PVAC was positioned consecutively at the ostia of each pulmonary vein (PV). The proper position was assessed before the first RF delivery at each PV by means of a hand-held injection (8-10 mL) of contrast medium (Optiray, Covidien Deutschland GmbH, Germany) diluted with saline in a $1: 1$ ratio through the FlexCath sheath, which was positioned close to the anticipated PV ostium. The saline infusion used for continuous flushing of the FlexCath sheath was interrupted for PV angiography and meticulous care was taken to eliminate any air bubbles in the sheath and in the syringe before contrast injections.

Once the optimal catheter position has been confirmed RF energy was applied in a 2:1 bipolar/ /unipolar mode for $60 \mathrm{~s}$, usually 3-4 times per PV. Simultaneous RF energy delivery to PVAC electrodes 1 (E1) and 10 (E10) was initiated only after careful fluoroscopic assessment of their positions when the inter-electrode distance appeared at least twice the fixed distance between two other adjacent electrodes. The electrode position was checked periodically during RF delivery. Any electrode pair that failed to reach a minimum of $50^{\circ} \mathrm{C}$ during RF delivery was switched off. RF delivery was also discontinued to electrodes after having reached the target temperature while delivering very low power (1-2 W).

The procedural endpoint was electrical isolation of all PVs, as confirmed by an entrance block evidenced by the disappearance of PV potentials is sinus rhythm, during coronary sinus pacing or during AF. No electrical cardioversion had been performed during the procedure or in the 
earlypost-ablation period before repeat cerebral MRI was completed. Heparin was discontinued at the end of the procedure with no protamine administered and the venous sheaths were removed 4-6 h later.

\section{TCD recording and evaluation}

Transcranial Doppler recording was performed throughout the whole period of LA access. The transducer was held in place by a proprietary headpiece supplied with the system. The MCAs were bilaterally insonated from trans-temporal windows by using a multi-frequency Doppler (Multi Dop T digital, DWL, QL software 2.8) which insonates simultaneously with frequencies of $2 \mathrm{MHz}$ and $2.5 \mathrm{MHz}$. The system is capable of the automatic online identification of true MESs with a sensitivity of $100 \%$ and a specificity of $99.3 \%$ and it also distinguishes between gaseous and solid emboli with a specificity of $96.5 \%[13,14]$. TCD parameter settings were as recommended by the consensus criteria [15] and were kept constant during the procedures. The insonation depth was $45-55 \mathrm{~mm}$, the sample volume was $8 \mathrm{~mm}$, and the power was 60-100 mW. MES counts were collected and evaluated separately during the different stages of the procedure as follows:

- trans-septal puncture: the 30 -s period after crossing the interatrial septum with the transseptal needle;

- PV angiography: contrast injection through the FlexCath trans-septal sheath. MESs were counted until they gradually decreased and disappeared after a marked burst, which usually followed the bolus within 5-10 s;

- energy delivery: from the start until $15 \mathrm{~s}$ after termination of energy delivery;

- the remainder of the procedure: that part of LA access period during which none of the aforementioned maneuvers were performed.

As bilateral recording was technically not possible in all patients, MES counts were calculated as mean MES count per patient, using either the mean of the bilateral counts when both sides were measured, or the unilateral data when only one side was available.

\section{Cerebral MRI}

Cerebral MRI was performed the day before and within $48 \mathrm{~h}$ after the procedure using a GE Sigma Excite 1.5 T (GE Medical Systems, Milwaukee, USA) scanner and an 8-channel phased array head coil. A routine brain imaging protocol was performed, including anaxial diffusion-weighted
(DWI) single-shot spin echo, echo-planar sequence [diffusion gradient b-values of 0 and $1000 \mathrm{~s} / \mathrm{mm}^{2}$, repetition time (TR): $8700 \mathrm{~ms}$, echo time (TE): 104 ms, slice thickness: $5 \mathrm{~mm}$, matrix: $128 \times 128$ ], an axial T2-weighted fast spin echo (TR: $5640 \mathrm{~ms}$, TE: $95 \mathrm{~ms}$, slice thickness: $3 \mathrm{~mm}$, matrix: $320 \times 256$ ), a sagittal T1-weighted spin echo (TR: $480 \mathrm{~ms}$, TE: $12 \mathrm{~ms}$, slice thickness: $3 \mathrm{~mm}$, matrix: $256 \times 160$ ) and a coronal fluid attenuated inversion recovery (FLAIR) [TR: $8002 \mathrm{~ms}$, TE: $130 \mathrm{~ms}$, slice thickness: $4 \mathrm{~mm}$, matrix: $320 \times 160$ ] sequence. In addition, a 25 directions DWI sequence was performed for advanced diffusion mapping.

DW MRI data were analyzed by two radiology experts blinded to the clinical status of the patients. The pre-procedure MRI was compared with the post-procedure MRI to identify any new procedure-related acute cerebral lesion defined as a focal hyper-intense area detected on the DWI confirmed by apparent diffusion coefficient mapping to rule out a shine-through artifact. The size and localization of focal diffusion abnormalities were analyzed. For patients who were positive for a new DWI lesion in the post-procedure MRI, another follow-up MRI was performed 3-month post-ablation.

\section{Statistical analysis}

Complete data sets for all patients were collected, including the results of intra-procedural TCD monitoring, pre- and post-ablation DW MRI. Patient and procedural characteristics were evaluated by using means and standard deviation (SD) or medians and interquartile range (IQR) for continuous variables accordingly, and counts and percentages for categorical variables. Groups of patients with and without DW MRI lesions were descriptively compared in terms of MES counts using Wilcoxon's rank-sum test. Logistic regression was used to evaluate the adjusted relationship between MES count and lesion development. Age was used as an apriori adjustment factor. Other potential explanatory variables were assessed for inclusion in the model. To improve distribution normality, age was cubic transformed, and the total MES count per MCA was natural log transformed. As curvature was observed in the relationship, a squared term for the log MES count was also used. Interactions were detected between the log MES count and age cubed, and between the squared log MES count and age cubed, and were therefore, included in the model. The effects of the MES count were expressed by comparing the odds for lesion development at a MES count of 1500 
(a value close to the sample $75^{\text {th }}$ percentile) to that at a count of 1100 (close to the sample median) in terms of odds ratios and $95 \%$ confidence intervals, specifically for levels across the range of age within which lesion development was observed. Model checking was based on the Hosmer-Lemeshow goodness of fit test.

\section{Results}

\section{Patient and procedural characteristics}

A total of 36 consecutive patients who underwent PVI with the PVAC and the GENius 14.4 generator gave consent to pre- and post-ablation cerebral MRI and to intra-procedural TCD monitoring. Two patients withdrew their consents from post-ablation MRI. TCD recording of MESs with sufficient quality could not be achieved in 7 , thereby the full data sets of 27 patients were available for analysis (Table 1).

A total of 108 PVs were successfully isolated. The mean (SD) procedure and fluoroscopy times were 106 (27.1) min and 23 (10), respectively. Procedural complications included 1 case of pericardial tamponade which required pericardiocentesis. Clinical stroke did not occur in any of the patients during or within 1 month after the ablation.

\section{TCD and MRI results}

The median (IQR) MES count per patient was 1151 (584). The highest rate of micro-embolization in any stage of the procedure was recorded during $\mathrm{RF}$ delivery (Fig. 1) and the majority of the MESs were gaseous during all phases of data collection (Fig. 2).

New SCI on DW MRI after PVI was demonstrated in $6(22 \%)$ patients: a single lesion in 2, two lesions in 3 , and three lesions in 1 case (Table 2). The average (SD) lesion diameter was 7.05 (2.9) $\mathrm{mm}(4.1-13.4 \mathrm{~mm})$. The majority of these lesions had disappeared by the 3-month follow-up: 1 patient had only a single lesion still detectable with a significant size reduction, and no new lesion as compared with the post-ablation image was demonstrated in any patient.

Several clinical parameters were investigated as possible predictors for SCI, including age, gender, LA diameter, left ventricular ejection fraction, $\mathrm{CHA}_{2} \mathrm{DS}_{2}$-VASC score, lacunary infarction on the preablation MRI, the total energy delivery time, the number of contrast injections during theprocedure, the percentage of concomitant use of E1/E10 and the mean intra-procedural ACT (Table 3). There was no significant difference among baseline clini-
Table 1. Baseline clinical and procedural parameters of the study population $(n=27)$.

\begin{tabular}{lc}
\hline Parameter & $\begin{array}{c}\text { Median (IQR) or } \\
\text { mean } \pm \text { SD }\end{array}$ \\
\hline Age [years] & $64(13.23)$ \\
Male/Female & $21 / 6$ \\
LA diameter [mm] & $41.88 \pm 4.39$ \\
LVEF [\%] & $56.77 \pm 3.61$ \\
CHADS ${ }_{2}$-Vasc: & \\
0 & 2 \\
1 & 10 \\
$\geq 2$ & 15 \\
Lacunary infarction present & $16(59.2 \%)$ \\
on pre-ablation MRI & $1001.63 \pm 280.47$ \\
Total energy delivery time [s] & $5 \pm 3$ \\
Number of contrast injections & $34.2 \pm 21.45$ \\
E1-E10 concomitantly active & $341(52)$ \\
[\% of the EDs] & \\
ACT [s] &
\end{tabular}

IQR - interquartile range; LA — left atrium; LVEF — left ventricular ejec-tion fraction; MRI — magnetic resonance imaging; ACT — activated clotting time; SD — standard deviation; EDs - energy deliveries

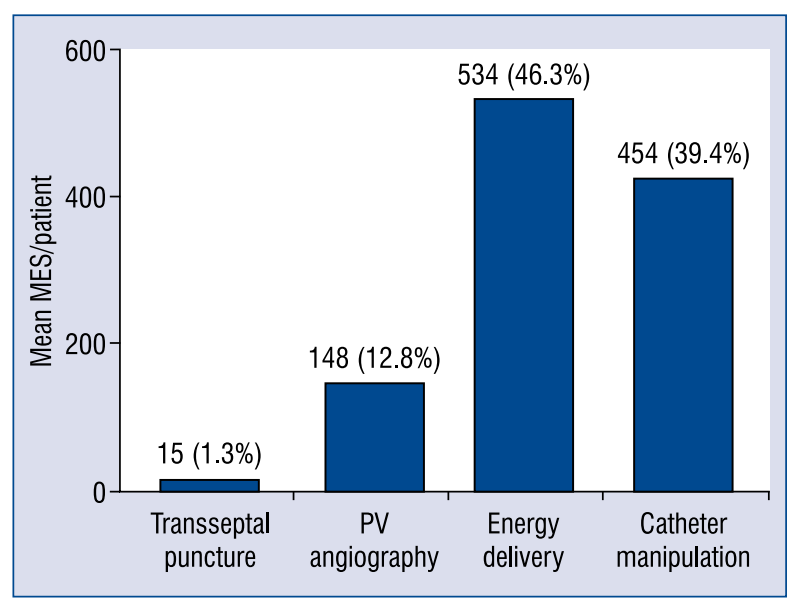

Figure 1. Total micro-embolic signal (MES) counts during different stages of the procedure. Figure depicting the number of MESs recorded during different stages of the pulmonary vein (PV) isolation. The highest rate of micro-embolization in any stage of the procedure was recorded during radiofrequency delivery.

cal parameters between patients with and without new SCI.

\section{Association between micro-embolization and MRI lesions}

Comparative analysis between TCD and MRI results are shown in Table 4 . The total median 


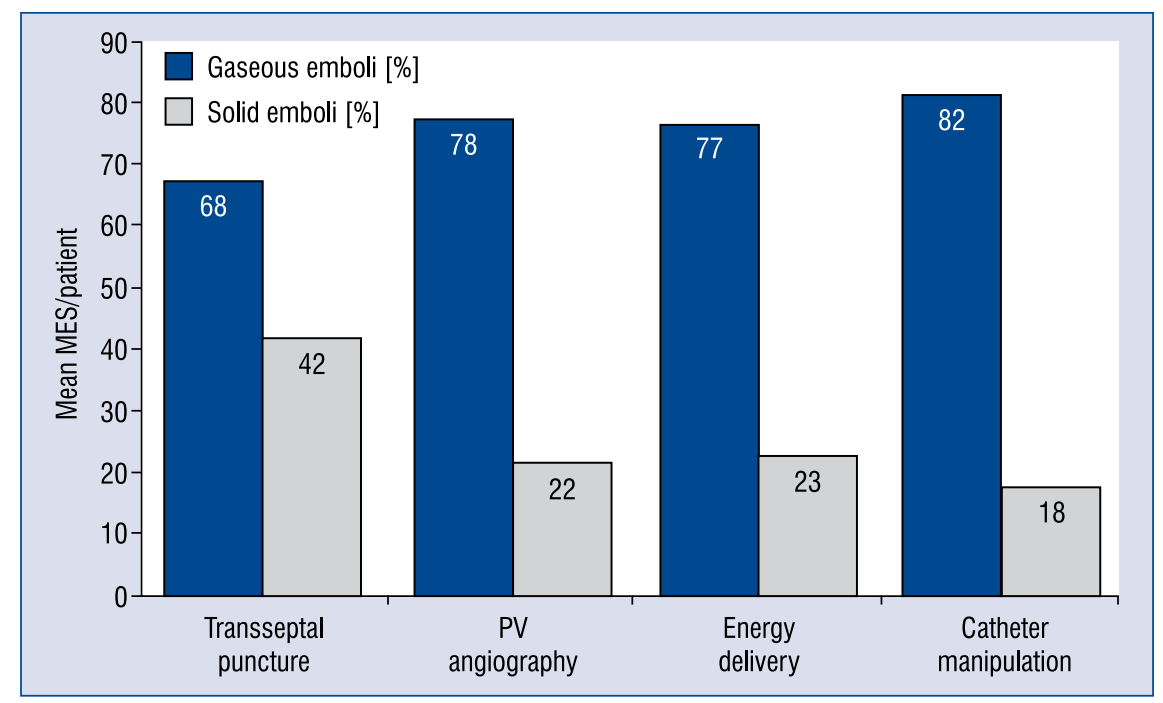

Figure 2. Ratio of solid versus gaseous emboli during different stages of the procedure. Figure illustrating the percentage of solid and gaseous micro-emboli detected during pulmonary vein (PV) isolation. The majority of the micro-embolic signal (MES) were gaseous during all phases of data collection.

Table 2. Magnetic resonance imaging (MRI) lesion characteristics after pulmonary vein isolation.

\begin{tabular}{cccccc}
\hline Patient & $\begin{array}{c}\text { No. of } \\
\text { lesions }\end{array}$ & $\begin{array}{c}\text { Lesion size } \\
{[\mathrm{mm}]}\end{array}$ & $\begin{array}{c}\text { Presence } \\
\text { on DWI }\end{array}$ & $\begin{array}{c}\text { Presence } \\
\text { on FLAIR }\end{array}$ & $\begin{array}{c}\text { Persistent lesion on } \\
\text { 3-month control MRI }\end{array}$ \\
\hline 1 & 2 & 8.5 & Yes & Yes & No \\
2 & 9.5 & Yes & Yes & No \\
3 & 1 & 4.6 & Yes & Yes & No \\
& 3 & 4.4 & Yes & No & No \\
& & 4.9 & Yes & No & No \\
4 & 2 & 6.6 & Yes & No & No \\
& 2 & 4.7 & Yes & No & No \\
5 & 2.6 & Yes & Yes & No \\
& & 8.3 & Yes & Yes & No \\
\hline
\end{tabular}

DWI — diffusion-weighted image sequence; FLAIR — fluid-attenuated inversion recovery sequence

MES count (IQR) during the procedures among the patients who demonstrated new acute MRI lesions was 1642.25 (912) as compared with 1019 (529) recorded in those who had no new lesions, the difference did not reach the level of statistical significance at this patient cohort size $(\mathrm{p}=0.129)$. However, the MES count per patient recorded during PV angiography was significantly higher in the patients with versus those without new brain lesions: 257 (249) vs. 110 (71), respectively ( $\mathrm{p}=$ $=0.0009$ ). Further, the multivariate logistic regression results demonstrated that the total MES count detected during the entire procedure was predic- tive of silent cerebral lesions at an advanced age: patients aged 68 years or older were significantly more likely, in a progressive manner to acquire new brain lesions if the number of MESs was high (Table 5).

\section{Discussion}

According to available research, this is the first study that has assessed the relationship between post-ablation SCI on DW MRI and the number of micro-emboli detected by TCD during PVI. A higher total MES count recorded during 
Table 3. Clinical and procedural parameters in patients with or without new silent ischemic lesions.

\begin{tabular}{|c|c|c|c|}
\hline & $\begin{array}{l}\text { Patients with } \\
\text { DW MRI lesions }(n=6)\end{array}$ & $\begin{array}{l}\text { Patients without } \\
\text { DW MRI lesions }(n=21)\end{array}$ & $\mathbf{P}$ \\
\hline Age [years]; median (IQR) & $63.7(12.3)$ & $65.5(7.1)$ & 0.22 \\
\hline Male/Female & $5 / 1$ & $16 / 5$ & 0.596 \\
\hline LA diameter $[\mathrm{mm}] ;$ median (IOR) & $41(4)$ & $42(6)$ & 0.452 \\
\hline LVEF [\%]; median (IQR) & $55(4)$ & $57(5)$ & 0.2 \\
\hline $\mathrm{CHA}_{2} \mathrm{DS}_{2}$-Vasc; median (IQR) & $2(1)$ & $2(2)$ & 0.513 \\
\hline $\begin{array}{l}\text { Lacunary infarction present } \\
\text { on pre-ablation MRI }\end{array}$ & $4(66.67 \%)$ & $13(61.9 \%)$ & 0.613 \\
\hline Total energy delivery time [s]; median (IQR) & $1045.3(263)$ & $978(382)$ & 0.64 \\
\hline Number of contrast injections; median (IQR) & $5.5(5)$ & $4(1)$ & 0.179 \\
\hline $\begin{array}{l}\text { E1-E10 concomitantly active (\% of the EDs); } \\
\text { median (IQR) }\end{array}$ & $21(10)$ & $36(30)$ & 0.121 \\
\hline ACT [s]; median (IQR) & $351.2(58)$ & $341(50)$ & 0.43 \\
\hline
\end{tabular}

IQR - interquartile range; DW MRI — diffusion-weighted magnetic resonance imaging; ACT — activated clotting time; LA — left atrium; LVEF - left ventricular ejection fraction; EDs - energy deliveries

Table 4. Transcranial Doppler results in patients with or without new lesions on post-ablation DW MRI.

\begin{tabular}{lccc}
\hline $\begin{array}{l}\text { MES count during different stages } \\
\text { of the procedure }\end{array}$ & $\begin{array}{c}\text { Patients with } \\
\text { DW MRI lesions } \\
\text { (n = 6) }\end{array}$ & $\begin{array}{c}\text { Patients without } \\
\text { DW MRI lesions } \\
\text { (n = 21) }\end{array}$ \\
\hline Total MES/MCA during transseptal puncture; median (IOR) & $11.5(11.5)$ & $4.75(7)$ & 0.759 \\
Total MES/MCA during PV angiography; median (IOR) & $257(249)$ & $110(71)$ & 0.0009 \\
Total MES/MCA during energy delivery; median (IOR) & $559(679.5)$ & $560.5(450.5)$ & 0.521 \\
Total MES/MCA during catheter manipulation; median (IOR) & $541.25(573)$ & $382(251)$ & 0.35 \\
Total MES/MCA during the whole procedure; median (IQR) & $1642.25(912)$ & $1019(529)$ & 0.129 \\
\hline
\end{tabular}

DW MRI — diffusion-weighted magnetic resonance imaging; MES — micro-embolic signal; MCA — middle cerebral artery; IQR - interquartile range; PV — pulmonary vein

Table 5. Logistic regression effect estimates for a contrast of 1500 vs. 1100 in total micro-embolic signal count on the odds of magnetic resonance imaging lesion development across the observed range of age levels.

\begin{tabular}{lccc}
\hline Age & OR & $95 \% \mathrm{CI}$ & $\mathbf{P}$ \\
\hline 54 & 0.15 & $0.02-1.35$ & 0.0904 \\
56 & 0.22 & $0.03-1.46$ & 0.1175 \\
58 & 0.34 & $0.07-1.62$ & 0.1779 \\
60 & 0.55 & $0.16-1.90$ & 0.3465 \\
62 & 0.92 & $0.34-2.47$ & 0.8677 \\
64 & 1.58 & $0.62-4.03$ & 0.3389 \\
66 & 2.81 & $0.90-8.79$ & 0.0759 \\
68 & 5.18 & $1.12-24.04$ & 0.0356 \\
70 & 9.92 & $1.29-76.05$ & 0.0273 \\
72 & 19.72 & $1.45-268.13$ & 0.0251 \\
\hline
\end{tabular}

$\mathrm{OR}$ - odds ratio; $\mathrm{Cl}$ - confidence interval the overall LA access time in patients with a new lesion on DW MRI (median: 1642 vs. 1019) was demonstrated, though the difference did not reach the level of statistical significance as there were a limited number of patients with positive MRI findings. Nonetheless, the relationship between the total MES count and SCI among the older patients was significant, suggesting that not only a micro-embolic load to the brain, but also the status of the cerebral vasculature might be relevant in lesion formation. Reduction of micro-embolus generation to the lowest possible level therefore seems especially important in the elderly, and it should be considered when selecting the ablation technique in this patient cohort. Further, additional risk of SCI has to be considered in these patients.

The MES count recorded during PV angiography proved to be a significant predictor of new cerebral lesions in this study. As the number of 
micro-emboli recorded during PV angiography comprises only a relatively small fraction of the total MES count recorded throughout the entire procedure, the strong correlation with SCI was somewhat unexpected. Although the majority are gaseous, similarly to those recorded during other phases of the procedure, the physical characteristics of MESs generated during contrast injections may differ. Moreover, PV angiography involves the injection of rapid boluses of contrast material into systemic circulation, and micro-emboli, although relatively small in number, are thereby, introduced within a very short time. It may be speculated that, besides the number of micro-emboli, the intensity of these micro-embolic showers reaching the brain circulation might also be an important determinant of lesion formation.

The present results are in line with recent data published from the Mayo Clinic. In an elegant in vivo pig model Takami et al. [16] performed different catheter, sheath manipulations and ablations while measuring micro-embolization using intracardiac echocardiography, Carotid Doppler, and an extra-corporal circulation loop which included a microbubble counter and blood filters to collect particulas. Significant microbubble formation was demonstrated on ICE, Carotid Doppler and in the loop during PV angiography and sheath flushing at a high flow, while micro-particles were mostly found during catheter dragging and after a steam pop. The present findings in patients appear to support these experimental data and may well have important practical implications as around half of the ablation centers worldwide [17] routinely perform PV angiography during PVI.

Importantly, the definition presented here of SCI was any new finding concerning the DW sequence with or without FLAIR positivity. This is different from the definitions used in most other studies [1, 2, 6, 7]. This more sensitive criterion was chosen to enable consideration of any detectable brain ischemia for comparison with MES data. FLAIR positivity was found in 5 of 6 patients and in 6 of 11 lesions in our study, and the majority of the lesions had resolved by the 3-month follow-up.

Although the aim of this research was to assess the relationship between TCD and DW MRI findings and not to evaluate the safety of phased RF ablation, it is noteworthy that lower incidences of SCI have been found in recent multi-center studies $[18,19]$. This favorable trend has been attributed to procedural modifications of phased RF ablation based on the results of clinical and preclinical stud- ies $[20,21]$. RF delivery to electrodes 1 and 10 getting close to each other due to a wedge position of the PVAC loop has been identified as the most significant contributor to embolus generation due to high current density, therefore simultaneous energization of these electrodes was prohibited in these trials and electrode 10 was even being removed from the novel PVAC Gold. Concomitant $\mathrm{RF}$ application on poles 1 and 10 was however not a priori excluded in the present study, and only when the inter-electrode distance on fluoroscopy was considered unsafe. Nonetheless, this more "liberal" practice did not contribute to a higher incidence of SCI lesions in this study.

The importance of SCI after AF ablation remains unclear. The concern that these lesions might be related to cognitive decline is supported by limited data [22, 23]. It has also been suggested that these lesions might be viewed as surrogates for the clinical stroke risk and are useful for an assessment of the risk involved with different ablation techniques and procedural changes [24]. The present data does suggest that TCD detection during LA ablation might improve our understanding of the micro-embolization process during these procedures.

\section{Limitations of the study}

The most important limitation of this study is the relatively small number of patients, and especially the low number of those with a new lesion on DW MRI after ablation. This exemplifies the challenges of enrolling patients that require nonstandard of care MRIs and the technical difficulties of TCD examination during relatively longer procedures in some patients. Although these observations reduced the power of the statistical analysis, a strong relationship between SCI and the MES count recorded during PV angiography was still demonstrated. Importantly, PVI was performed using phased RF exclusively in this study. Future studies with similar design but with different ablation technologies (cryoballoon, focal irrigated RF) might therefore be of interest.

\section{Conclusions}

This study demonstrates a relationship between micro-embolization detected by TCD over the MCA and acute SCI on DW MRI after PVI with phased radiofrequency and the pulmonary vein ablation catheter. Higher numbers of micro-emboli detected during pulmonary vein angiography were predictive of positive postablation findings on DW MRI. 


\section{Acknowledgements}

This work was supported by grant from the $\mathrm{Na}-$ tional Research, Development and Innovation Fund (K109712) and by the GINOP-2.3.2-15-2016-00043 project co-financed by the European Union and the European Regonal Development Fund.

Conflict of interest: Edina Nagy-Baló: none, Mihran Martyrosian: none, Gábor Sándorfi: none, Orsolya Hajas: none, Levente Lánczi: none, Ervin Berényi: none, Lilla Ladányi: none, Alexandra Kiss: none, István Édes: none, Zoltán Csanádi: speaker honorarium and grant support from Medtronic Inc.

\section{References}

1. Schrickel JW, Lickfett L, Lewalter T, et al. Incidence and predictors of silent cerebral embolism during pulmonary vein catheter ablation for atrial fibrillation. Europace. 2010; 12(1): 52-57, doi: 10.1093/europace/eup350, indexed in Pubmed: 19933517.

2. Gaita F, Caponi D, Pianelli M, et al. Radiofrequency catheter ablation of atrial fibrillation: a cause of silent thromboembolism? Magnetic resonance imaging assessment of cerebral thromboembolism in patients undergoing ablation of atrial fibrillation. Circulation. 2010; 122(17): 1667-1673, doi: 10.1161/CIRCULATIONAHA.110.937953, indexed in Pubmed: 20937975.

3. Neumann T, Kuniss M, Conradi G, et al. MEDAFI-Trial (Microembolization during ablation of atrial fibrillation): comparison of pulmonary vein isolation using cryoballoon technique vs. radiofrequency energy. Europace. 2011; 13(1): 37-44, doi: 10.1093/ europace/euq303, indexed in Pubmed: 20829189.

4. Lickfett L, Hackenbroch M, Lewalter T, et al. Cerebral diffusion-weighted magnetic resonance imaging: a tool to monitor the thrombogenicity of left atrial catheter ablation. J Cardiovasc Electrophysiol. 2006; 17(1): 1-7, doi: 10.1111/j.15408167.2005.00279.x, indexed in Pubmed: 16426390.

5. Herrera Siklódy C, Deneke T, Hocini M, et al. Incidence of asymptomatic intracranial embolic events after pulmonary vein isolation: comparison of different atrial fibrillation ablation technologies in a multicenter study. J Am Coll Cardiol. 2011; 58(7): 681-688, doi: 10.1016/j.jacc.2011.04.010, indexed in Pubmed: 21664090

6. Gaita F, Leclercq JF, Schumacher B, et al. Incidence of silent cerebral thromboembolic lesions after atrial fibrillation ablation may change according to technology used: comparison of irrigated radiofrequency, multipolar nonirrigated catheter and cryoballoon. J Cardiovasc Electrophysiol. 2011; 22(9): 961-968, doi: 10.1111/j.1540-8167.2011.02050.x, indexed in Pubmed: 21453372.

7. Deneke T, Shin DI, Balta O, et al. Postablation asymptomatic cerebral lesions: long--term follow-up using magnetic resonance imaging. Heart Rhythm. 2011; 8(11): 1705-1711, doi: 10.1016/j. hrthm.2011.06.030, indexed in Pubmed: 21726519.

8. Kilicaslan F, Verma A, Saad E, et al. Transcranial Doppler detection of microembolic signals during pulmonary vein antrum isolation: implications for titration of radiofrequency energy. J Cardiovasc Electrophysiol. 2006; 17(5): 495-501, doi: 10.1111/j.15408167.2006.00451.x, indexed in Pubmed: 16684021.

9. Nagy-Baló E, Tint D, Clemens M, et al. Transcranial measurement of cerebral microembolic signals during pulmonary vein isolation: a comparison of two ablation techniques. Circ Arrhythm Electrophysiol. 2013; 6(3): 473-480, doi: 10.1161/CIRCEP.112.971747, indexed in Pubmed: 23580744.

10. Kiss A, Nagy-Baló E, Sándorfi G, et al. Cerebral microembolization during atrial fibrillation ablation: comparison of different single-shot ablation techniques. Int J Cardiol. 2014; 174(2): 276-281, doi: 10.1016/j.ijcard.2014.03.175, indexed in Pubmed: 24767748.
11. Nagy-Balo E, Kiss A, Condie C, et al. Predictors of cerebral microembolization during phased radiofrequency ablation of atrial fibrillation: analysis of biophysical parameters from the ablation generator. Heart Rhythm. 2014; 11(6): 977-983, doi: 10.1016/j. hrthm.2014.03.018, indexed in Pubmed: 24637195.

12. Nagy-Balo E, Kiss A, Condie C, et al. Predictors of cerebral microembolization during phased radiofrequency ablation of atrial fibrillation: role of the ongoing rhythm and the site of energy delivery. Pacing Clin Electrophysiol. 2014; 37(11): 1436-1441, doi: 10.1111/pace.12454, indexed in Pubmed: 25130865.

13. Russell D, Brucher R. Online automatic discrimination between solid and gaseous cerebral microemboli with the first multifrequency transcranial Doppler. Stroke. 2002; 33(8): 1975-1980, doi: 10.1161/01.str.0000022809.46400.4b.

14. Markus HS, Punter M. Can transcranial Doppler discriminate between solid and gaseous microemboli? Assessment of a dualfrequency transducer system. Stroke. 2005; 36(8): 1731-1734, doi: 10.1161/01.STR.0000173399.20127.b3, indexed in Pubmed: 16020767 .

15. Ringelstein EB, Droste DW, Babikian VL, et al. Consensus on microembolus detection by TCD. International Consensus Group on Microembolus Detection. Stroke. 1998; 29(3): 725-729, indexed in Pubmed: 9506619.

16. Takami M, Lehmann HI, Parker KD, et al. Effect of left atrial ablation process and strategy on microemboli formation during irrigated radiofrequency catheter ablation in an in vivo model. Circ Arrhythm Electrophysiol. 2016; 9(1): e003226, doi: 10.1161/ CIRCEP.115.003226, indexed in Pubmed: 26763224.

17. Calkins H, Kuck K, Cappato R, et al. 2012 HRS/EHRA/ECAS expert consensus statement on catheter and surgical ablation of atrial fibrillation: recommendations for patient selection, procedural techniques, patient management and follow-up, definitions, endpoints, and research trial design. Heart Rhythm. 2012; 9(4): 632-696.e21, doi: 10.1016/j.hrthm.2011.12.016.

18. Verma A, Debruyne P, Nardi S, et al. ERACE Investigators. Evaluation and reduction of asymptomatic cerebral embolism in ablation of atrial fibrillation, but high prevalence of chronic silent infarction: results of the evaluation of reduction of asymptomatic cerebral embolism trial. Circ Arrhythm Electrophysiol. 2013; 6(5): 835-842, doi: 10.1161/CIRCEP.113.000612, indexed in Pubmed: 23983245.

19. De Greef Y, Dekker L, Boersma L, et al. PRECISION GOLD Investigators. Low rate of asymptomatic cerebral embolism and improved procedural efficiency with the novel pulmonary vein ablation catheter GOLD: results of the PRECISION GOLD trial. Europace. 2016; 18(5): 687-695, doi: 10.1093/europace/euv385, indexed in Pubmed: 26826134.

20. Wieczorek M, Lukat M, Hoeltgen R, et al. Investigation into causes of abnormal cerebral MRI findings following PVAC dutycycled, phased RF ablation of atrial fibrillation. J Cardiovasc Electrophysiol. 2013; 24(2): 121-128, doi: 10.1111/jce.12006, indexed in Pubmed: 23134483.

21. Haines DE, Stewart MT, Dahlberg S, et al. Microembolism and catheter ablation I: a comparison of irrigated radiofrequency and multielectrode-phased radiofrequency catheter ablation of pulmonary vein ostia. Circ Arrhythm Electrophysiol. 2013; 6(1): 16-22, doi: 10.1161/CIRCEP.111.973453, indexed in Pubmed: 23392585 .

22. Schwarz N, Kuniss M, Nedelmann M, et al. Neuropsychological decline after catheter ablation of atrial fibrillation. Heart Rhythm. 2010; 7(12): 1761-1767, doi: 10.1016/j.hrthm.2010.07.035, indexed in Pubmed: 20691284.

23. Medi C, Evered L, Silbert B, et al. Subtle post-procedural cognitive dysfunction after atrial fibrillation ablation. J Am Coll Cardiol. 2013; 62(6): 531-539, doi: 10.1016/j.jacc.2013.03.073, indexed in Pubmed: 23684686.

24. Csanadi Z, Nagy-Baló E, Danik S, et al. Cerebrovascular complications related to atrial fibrillation ablation and strategies for periprocedural stroke prevention. Card Electrophysiol Clin. 2014; 6(1): 111-123, doi: 10.1016/j.ccep.2013.10.003, indexed in Pubmed: 27063826. 